\title{
Malignant Edema in Horse by Clostridium perfringens Type A
}

\author{
Jair Alves Ferreira Junior', Rodrigo Otávio Silveira Silva², Francisco Carlos Faria Lobato², \\ Antônio Raphael Teixeira $\mathrm{Neto}^{3}$, Karla Alvarenga Nascimento', \\ Juliana Targino Silva Almeida e Macêdo' \& Pedro Miguel Ocampos Pedroso'
}

\begin{abstract}
Background: Malignant edema is one of the terms used to designate severe necrotizing syndromes in soft tissues by Clostridium spp. which are potentially fatal in farm animals. These species are responsible for myonecrosis, belonging to the group of histotoxic clostridia, and may also culminate in toxemia with the worsening of the lesions. These clostridia and their spores require a gateway such as wounds on mucous membranes or skin, which may occur due to shear, tail cut, injuries during delivery, castration or injections by contaminated needles. This report aims to describe the clinicalpathological findings of a case of malignant edema caused by Clostridium perfringens type $\mathrm{A}$ in an equine.

Case: A female equine, undefined breed, used as traction animal, had a history of abdominal pain. According to the requisitioning veterinary, the tutor reused needles for medication. On palpation, a compact mass was noticed in the pelvic flexure, as well as edema on the region of head and neck with crackling areas. After surgical intervention for compactation correction, the animal did not show anesthetic recovery and was submited to euthanasia. Tissue samples were collected, fixed in 10\% buffered formalin solution, routinely processed for histopathology and stained with hematoxylin and eosin and Gram stain. Samples of serous-sanguineous edema fluid and fragments of the abdominal muscles and neck were collected. The samples were kept under refrigeration and sent for microbiological culture. Necropsy showed the subcutaneous region of the pectoral was markedly gelatinous and yellowish (edema) and subcutaneous emphysema characterized by accumulation of serous-sanguineous fluid and gas bubbles. In microscopy, we verified fibrous-haemorrhagic, emphysematous, suppurative and diffuse superficial histiocytic necrotizing cellulitis and myositis. Gram-positive bacillary aggregates were observed in spleen and subcutaneous sections. Colony suggestive of Clostridium perfringens were submitted to PCR for confirmation of identity followed by genotyping. Lastly, C. perfringens type A was isolated from the muscle fragments and serosanguinolent liquid collected.

Discussion: In the present study, the diagnosis of malignant edema caused by Clostridium perfringens type A in an equine was confirmed by the clinical-pathological findings added to the isolation and genotyping of the agent from fragments of injured muscle. Although $C$. perfringens has been reported in cases of clostridial myositis in horses in the United States, in Brazil there are only reports of sporadic cases associated with C. septicum, C. novyi and C. chauvoei in this species. In histotoxic cases by $C$. perfringens, alpha toxin is known to be the main virulence factor involved, causing destruction of the phospholipid membrane of erythrocytes, endothelial cells, leukocytes and muscle fibers. As consequence, there is an increase in the vascular permeability of capillaries and, with the spread of the infection, there is production of inflammatory edema with serosanguineous exudate and gas. Typically, anatomopathological findings are characterized by increased volume of the affected region associated with edema with bloody fluid and rancid odor, gas bubbles in the subcutaneous tissue, and fasciae associated or not with necrotic myositis. It is assumed that a contaminated needle injection, performed during the treatment of colic symptoms on the property has been the gateway to infection. The clinical course of clostridial infection in horses is considered acute, ranging from 24 to $48 \mathrm{~h}$. In this case, was observed the animal with signs of cervical and pectoral edema still in the property, a day before euthanasia. This is the first study to confirm Clostridium perfringens as a cause of gas gangrene in horses in Brazil.
\end{abstract}

Keywords: myonecrosis, clostridiosis, emphysematous, pathology, PCR. 


\section{INTRODUCTION}

Malignant edema is one of the terms used by several authors to designate severe necrotizing syndromes in soft tissues by Clostridium spp. which are potentially fatal in horses. Other terms such as clostridial myositis and clostridial cellulitis are considered synonyms by some authors [4]. Malignant edema is frequently observed in cattle, sheep and goats, with a higher susceptibility, in addition to equines and pigs to a lesser extent [7].

Among the main causative bacteria are Clostridium septicum, C. perfringens [4,7], C. novyi type A [1] and C. novyi type B, C. sordelli [9] and C. chauvoei, [2]. These species are responsible for myonecrosis, belonging to the group of histotoxic clostridia, and may also culminate in toxemia with the worsening of the lesions [10]. These clostridia and their spores require a gateway such as wounds on mucous membranes or skin, which may occur due to shear, tail cut, injuries during delivery, castration or injections by contaminated needles $[2,9,10]$.

Malignant edema cases in horses are rare, with only a few reports in the literature. In Brazil, there are only three reports associated with $C$. chauvoei, $C$. septicum and $C$. novyi $[1,2,7]$. Thus, the objective of the present study is to describe for the first time the clinical-pathological findings of a case of malignant edema caused by $C$. perfringens type $\mathrm{A}$ in an equine.

\section{CASE}

A female equine used as traction animal, of undefined breed, was forwarded to the veterinary hospital with history of abdominal pain and increase of volume of the region of head and neck. The information obtained that the mare was treated with medication administered parenterally with syringe and a used needle. The tutor did not provide data about the medication previously administered. During rectal palpation, a compact mass was noticed in the pelvic flexure. The clinical examination also showed that the animal presented bilateral edema in the head, neck, thorax and abdomen regions, with areas of crackling. After surgical intervention to correct the compaction, the animal did not showed anesthesia recovery and was submitted to euthanasia.

During necropsy the subcutaneous region of the pectoral region was markedly gelatinous and yellowish (edema). In the right thoracic and cervical antimer, there was a focally extensive area of the subcutaneous tissue, starting from the neck to the ileum wing, markedly irregular, swollen, yellowish with red areas, with a spumous appearance between the muscular layers (emphysema). The musculature involved was markedly friable, roseate, opaque and imbibed by serosanguinolent fluid (Figure 1A). By opening the abdominal cavity, there was a moderate amount of reddish serous fluid and a linear suture of approximately $8.0 \mathrm{~cm}$ was found in the pelvic flexure. Tissue samples were collected, fixed in $10 \%$ buffered formalin solution, routinely processed for histopathology and stained with hematoxylin and eosin and Gram stain $^{1}$. Samples of serous-sanguineous edema fluid were collected from subcutaneous and intramuscular region, as well as fragments of the abdominal muscles and neck. The samples were kept under refrigeration and sent for microbiological culture.

Microscopically, there was in the skeletal muscle and subcutaneous a marked infiltration of neutrophils, macrophages and a smaller amount of lymphocytes associated with fibrillar eosinophilic material (fibrin), nuclei remnants in karyiorrexia together with cell debris (necrosis) and vacuoles that expanded the subcutaneous tissue and the perimysium. Multifocal areas of haemorrhage were also observed permeating the infiltrate. The superficial muscle bundles were hypereosinophilic and often replaced by spumous macrophages and neutrophils. In the deeper musculature there were multifocal areas of separation in the myofibers by uncolored gaseous bubble strands of about $50 \mu \mathrm{m}$ in diameter (Figure 1B).

In one of the fragments, the subcutaneous tissue was expanded by fibrin and the connective tissue was loosely arranged (edema) [Figure 1C]. Subsequently in the spleen, multifocal areas of necrosis were observed in the red pulp, which center contained Gram-positive basophilic bacillary bacterial aggregates evidenced by the Gram staining technique. Gram-positive bacilli were also visualized in subcutaneous sections (Figure 1D).

In microbiological culture, clinical specimens were grown on Muller-Hinton agar $^{2}$ supplemented with 5\% sheep blood in aero and anaerobiosis. Colony suggestive of $C$. perfringens were submitted to PCR for confirmation of identity [8] followed by genotyping [12]. Lastly, C. perfringens type A was isolated from the muscle fragments and serosanguinolent liquid collected. 


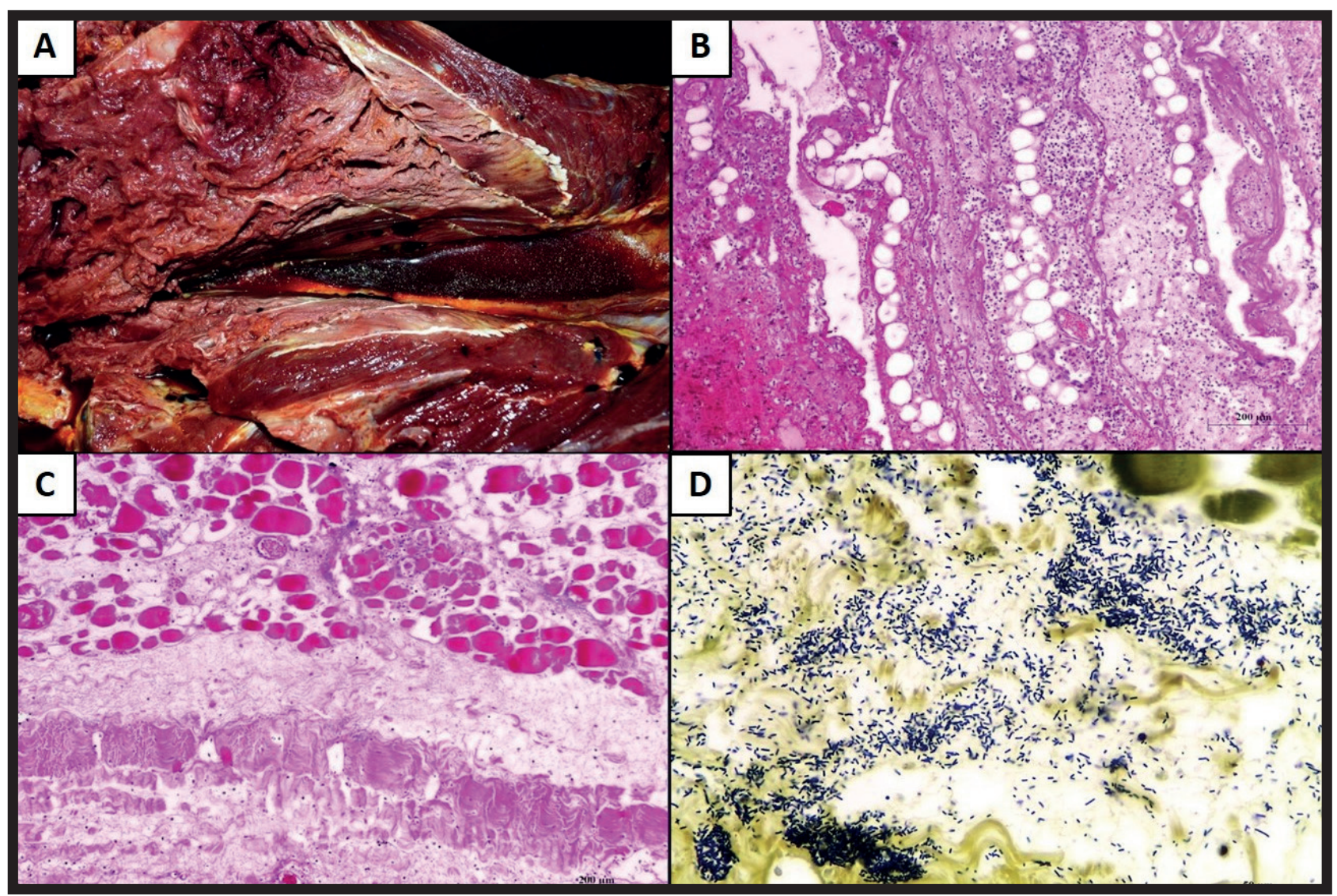

Figure 1. Malignant edema in horse by Clostridium perfringens type A. A- Severely friable cervical muscle from where serosanguinolent liquid flowed to the cut. B- In the subcutaneous, there is a marked infiltration of macrophage, neutrophils and fibrin associated with vacuoles [H\&E; obj.10x]. C- Subcutaneous: note tissue expanded by fibrin and connective tissue by loose-fitting arrangement (edema) [H\&E; obj.10x]. D- Subcutaneous and musculature evaluated bacterial evidence as Gram-positive bacilli [Gram; obj.10x].

\section{DISCUSSION}

As an etiologic agent of malignant edema, $C$. perfringens is recognized for its high occurrence in humans $[10,11]$. In the present study, the diagnosis of malignant edema caused by $C$. perfringens type A in an equine was confirmed by the clinical-pathological findings added to the isolation and genotyping of the agent from fragments of injured muscle. Although $C$. perfringens has been reported in cases of clostridial myositis in horses in the United States [4], in Brazil there are only reports of sporadic cases associated with $C$. septicum, $C$. novyi and $C$. chauvoei in this species [1,7]. Thus, this is the first report of gas gangrene by $C$. perfringens in horses in Brazil. It is interesting to note that, despite the novelty in equines in Brazil, a recent study has revealed $C$. perfringens as one of the main agents involved in gas gangrene in ruminants in the country, suggesting that the importance of this agent may have been underestimated in different domestic species [5].

In histotoxic cases by $C$. perfringens, alpha toxin is known to be the main virulence factor involved
$[6,10]$, causing destruction of the phospholipid membrane of erythrocytes, endothelial cells, leukocytes and muscle fibers. As consequence, there is an increase in the vascular permeability of capillaries and, with the spread of the infection, there is production of inflammatory edema with serosanguinolous exudate and gas [3]. Typically, anatomopathological findings are characterized by increased volume of the affected region associated with edema with bloody fluid and rancid odor, gas bubbles in the subcutaneous tissue, and fasciae associated or not with necrotic myositis [2,7]. In the present case, a necrotizing fibrin-hemorrhagic cellulitis with a lower degree of myonecrosis, similar to the ones described by $C$. chauvoei and $C$. septicum in horses [2,7], was predominantly observed.

It is assumed that a contaminated needle injection, performed during the treatment of colic symptoms on the property has been the gateway to infection. The clinical course of clostridial infection in horses is considered acute, ranging from 24 to $48 \mathrm{~h}$ $[2,7,9]$. In this case, we observed the animal with signs 
of cervical and pectoral edema still in the property, a day before euthanasia. However, it is estimated that the clinical course is longer than $48 \mathrm{~h}$ due to the marked degree of inflammation and necrosis with macrophage involvement.

The detection of Clostridium perfringens points out that this agent may be related to cases of necrotic myositis in horses, as reported in previous studies in the USA [4]. This is the first study to confirm C. perfringens as a cause of gas gangrene in horses in Brazil.

\section{MANUFACTURERS}

${ }^{1}$ WCOR Corantes. São Paulo, SP, Brazil.

${ }^{2}$ Difco Laboratories Inc. Detroit, MI, USA.

Acknowledgments. The authors thank the veterinarian Florence Marie Berthier (EMATER-DF), the resident veterinarian Camila Osse (UnB) for the clinical and epidemiological information of the equine, and Estefânia Melgaço for assistance in collecting and sending the material.

Declaration of interest. The authors report no conflicts of interest. The authors alone are responsible for the content and writing of the paper.

\section{REFERENCES}

1 Farias L., Azevedo M.D.S., Trost M.E., De La Côrte F.D., Irigoyen L.F. \& Vargas A.C.D. 2014. Acute myonecrosis in horse caused by Clostridium novyi type A. Brazilian Journal of Microbiology. 45(1): 221-224.

2 Macêdo J.T.S.A., Pires P.S., Pinheiro E.E.G., Oliveira R.S., Silva R.O.S., Lobato F.C.F. \& Pedroso P.M.O. 2007. Edema maligno em equino causado por Clostridium chauvoei. Acta Scientiae Veterinariae. 41: 1-4.

3 Odendaal M.W. \& Kriek N.P.J. 2004. Clostridium septicum infections. In: Coetzer J.A.W. \& Tustin R.C. (Eds). Infectious Diseases of Livestock. Cape Town: Oxford University Press, pp.1869-1873.

4 Peek S.F., Semrad S.D. \& Perkins G.A. 2003. Clostridial myonecrosis in horses (37 cases 1985-2000). Equine Veterinary Journal. 35(1): 86-92.

5 Pires P.S., Ecco R., Silva R.O.S., Araújo M.R.D., Salvarani F.M., Heneine L.G.D. \& Lobato F.C.F. 2017. A retrospective study on the diagnosis of clostridial myonecrosis in ruminants in Brazil. Ciência Rural. 47(1): 1-5.

6 Popoff M.R. \& Bouvet P. 2013. Genetic characteristics of toxigenic Clostridia and toxin gene evolution. Toxicon. 75: 63-89.

7 Raymundo D.L., Pavarini S.P., Bezerra Junior P.S., Antoniassi N.A.B., Bandarra P.M., Bercht B.S. \& Driemeier D. 2010. Mionecrose aguda por Clostridium septicum em equinos. Pesquisa Veterinária Brasileira. 30(8): 637-640.

8 Ribeiro M.G., Silva R.O.S., Pires P.S., Martinho A.P.V., Lucas T.M., Teixeira A.I.P. \& Lobato F.C.F. 2012. Myonecrosis by Clostridium septicum in a dog, diagnosed by a new multiplex-PCR. Anaerobe. 18(5): 504-507.

9 Riet-Correa F. 2007. Edema maligno. In: Riet-Correa F., Schild A.L., Lemos R.A.A. \& Borges J.R. (Eds). Doenças de Ruminantes e Equídeos. 3.ed. v.1. Santa Maria: Pallotti, pp.286-288.

10 Silva R.O.S., Uzal F.A., Oliveira Jr. C.A.O. \& Lobato F.C.F. 2016. Gas gangrene (malignant edema). In: Uzal F., Prescott J., Songer G. \& Popoff M. (Eds). Clostridial Diseases of Animals. Ames: John Wiley \& Sons, pp.243-254.

11 Stevens D.L., Aldape M.J. \& Bryant A.E. 2012. Life-threatening clostridial infections. Anaerobe. 18(2): 254-259.

12 Vieira A.A.S., Guedes R.M.C., Salvarani F.M., Silva R.O.S., Assis R.A. \& Lobato F.C.F. 2008. Genotipagem de Clostridium perfringens isolados de leitões diarreicos. Arquivos do Instituto Biológico. 75(4): 513-516. 